\title{
Prediction Primary Radiation Shielding Wall Thickness with Artificial Neural Networks
}

\author{
A. AKKAŞ ${ }^{a}$, C. BAŞYIĞIT ${ }^{b}$ AND M. NeCIP KuRTARICI ${ }^{b}$ \\ ${ }^{a}$ Suleyman Demirel University, Teknoloji Fak İmalat Müh., Isparta, Turkey \\ ${ }^{b}$ Suleyman Demirel University, Teknik Egt. Fak. Yapı Egt. Bol., Isparta, Turkey
}

\begin{abstract}
In this study, wall thickness for using in primary radiation shielding was determined in different energy ranges using tenth value layer by artificial neural networks. Radiation energy values, tenth value layers and negative logarithm of transmission factor $(n)$ were selected as input parameters and wall shielding thickness values selected as output parameters. Consequently, developed artificial neural networks model outputs were compared with experimental results and it was seen that the results were harmonious.
\end{abstract}

DOI: 10.12693/APhysPolA.123.171

PACS: 28.41.Qb, 84.35.+i

\section{Introduction}

Radiation is an energy that exists everywhere. We cannot ignore the risk of radiation so we should take precautions to minimize the danger of radiation. One of the biggest concerns regarding the responsible use of radiation is protecting the public and workers from deleterious exposure to ionization radiation; because of this, safe exposure limits for both the general public and occupationally exposed individuals are appropriately regulated. Consistent with these regulatory limits, facilities in which radiation is generated and used are required to have appropriate shielding capabilities, as stipulated by the National Council on Radiation Protection and Measurements $[1,2]$.

The purpose of radiation shielding is to reduce the effective equivalent dose from a linear accelerator to a point outside the room to a sufficiently low level, one that is determined by individual states; this level is generally $0.01 \mathrm{mSv}$ per week for a controlled area [3].

In this study, wall thickness for using in primary radiation shielding was determined in different energy ranges using tenth value layer by artificial neural networks (ANN). Radiation energy values, tenth value layers (TVL) and negative logarithm of transmission factor $(n)$ were selected as input parameters and wall shielding thickness values selected as output parameters. Consequently, developed artificial neural networks model outputs were compared with experimental results.

\section{Artificial neural networks}

Artificial neural networks are computer systems developed with the aim of automatically performing capabilities such as generating, learning and exploring of new knowledge through learning which are characteristic of the human brain. This ability to perform with conventional programming methods is very difficult or impossible. Therefore, neural networks may be said to be discipline interested in adaptive information processing, improved for events which is very difficult or not possible to program $[4,5]$. ANN consists of three layers, namely input, hidden, and output layers; input layer neurons, hidden layer neurons, output layer neurons. A neuron consists of multiple inputs and a single output. The sum of the inputs and their weights lead to a summation operation as Eq. (1)

$$
\mathrm{NET}_{j}=\sum_{1}^{n} w_{i} x_{i},
$$

in which $w_{i j}$ is established weight, $x_{i j}$ is input value and $\mathrm{NET}_{j}$ is input to a node in layer $j$. The output of a neuron is decided by an activation function. There are a number of activation functions that can be used in ANNs such as step, sigmoid, threshold, linear etc. The sigmoid activation function, $f(x)$, which is commonly used because of its nonlinearity and its advantages of smooth and easily differentiation, and can be formulated mathematically as Eq. (2), Eq. (3):

$$
\begin{aligned}
& f_{x}=1 /[1+\exp (-x)], \\
& \operatorname{OUTPUT}_{j}=f\left(\mathrm{NET}_{j}\right)=1 /\left[1+\exp \left(-\mathrm{NET}_{j}\right)\right] .
\end{aligned}
$$

\section{Application and data}

Primary radiation is that radiation used to treat the patient. Though the typical treatment field size is generally less than $20 \times 20 \mathrm{~cm}^{2}$, for shielding purposes, the maximum field size of $40 \times 40 \mathrm{~cm}^{2}$ is always assumed (see section on barrier widths). The required barrier transmission factor, $B_{p}$, for primary radiation is given by Eq. (4):

$$
B_{p}=\frac{P d^{2}}{\mathrm{WUT}} .
$$

$P$ is the weekly designed dose limit derived from the annual limit appropriate for the type of space protected by the barrier and is determined by individual states; this level is generally $0.01 \mathrm{mSv}$ per week for a controlled area; $d$ is the distance from the target to the point of measurement $(\mathrm{m}) . W$ is the workload (Gy/week). $W$ value of the parameters in the following Eq. (5) is calculated using 


$$
W=40 \frac{\text { patients }}{\text { day }} 5 \frac{\text { fields }}{\text { patient }} 50 \frac{M U}{\text { field }} 1 \frac{\mathrm{cGy}}{M U} 5 \frac{\text { days }}{\text { week }} .
$$

$U$ is the use factor, $T$ is the occupancy factor. These transmission factors are converted to barrier thicknesses using Eq. (6):

$$
n=-\log _{10}\left(B_{p}\right) \mathrm{TVL} \text {, }
$$

where TVL is the appropriate tenth value layer for the radiation under consideration. For primary barriers it is not necessary to include the effects of secondary radiation. In this study concretes exposed to different radiation levels between $1 \mathrm{MeV}$ and $24 \mathrm{MeV}$ for the calculation of the thickness of the radiation intensity, radiation source, distance, and TVLs were used. Calculated for each radiation intensity until distance of $15 \mathrm{~m}$. 135 data were used in modeling. The training data sets consist of 100 data and the testing data sets consist of 35 data in the model. ANN model the values of input parameters and output parameters have different units, due to these values are have been made dimensionless with Eq. 7. ANN model the values of input parameters and output parameters of each of the different units, these values are due to their Eq. (7) and have been made dimensionless

$$
F=\left(F_{i}-F_{\min }\right) /\left(F_{\max }-F_{\min }\right) \text {, }
$$

where $F$ is the standardized value of the $F_{i} ; F_{\max }$ and $F_{\text {min }}$ are the maximum and minimum values in meusurements. In ANN model for each of five different values of the hidden layer there were used 10 different formulations. Number of neurons in hidden layer was determined with the best compliance with the results. For determining the best ANN model, the number of iterations for each model is limited to 10000 . In this study, the multilayer feed-forward ANN model was used to adjust the weights of the error back propagation algorithm is used. Model and model's both training and test values and root mean square error (RMSE) values are given in Table.

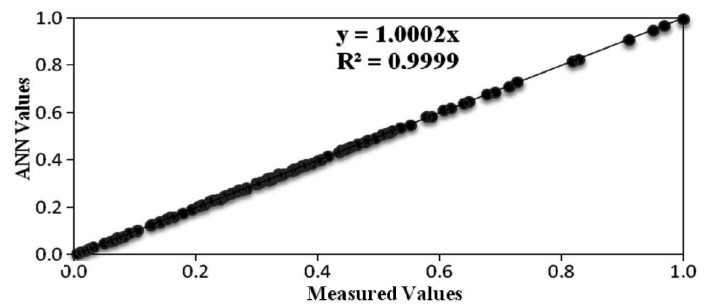

Fig. 1. Comparison of training and ANN model $(3,4,1)$ data sets.

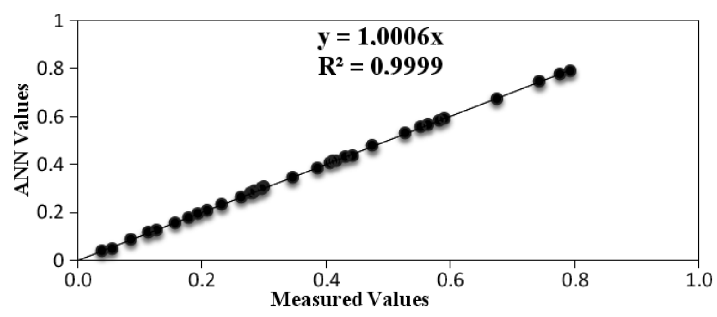

Fig. 2. Comparison of test and ANN model $(3,4,1)$ data sets.
As seen from Table, the best network architectures for $\operatorname{ANN}(3,4,1), \operatorname{ANN}(3,1,1), \operatorname{ANN}(3,2,1), \operatorname{ANN}(3,3,1)$, $\operatorname{ANN}(3,5,1)$ models are determined. Also, $R^{2}$ values of ANN $(3,4,1)$ were 0.99 and 0.99 for training and testing data sets,respectively (Figs.1 and 2). Considering ANN models, $\operatorname{ANN}(3,4,1)$ model has the best $R^{2}$ and the lowest RMSE value.

The $R^{2}$ and RMSE values of ANN models.

TABLE

\begin{tabular}{c|c|c|c|c}
\hline \hline \multirow{2}{*}{ ANN models } & \multicolumn{2}{|c|}{ Training data set } & \multicolumn{2}{c}{ Testing data set } \\
\cline { 2 - 5 } & Function & $R^{2}$ & $R^{2}$ & RMSE \\
\hline ANN(3,4,1) & Tansig4 & 0.99 & 0.99 & 1.04 \\
ANN $(3,1,1)$ & Logsig2 & 0.99 & 0.97 & 1.5 \\
ANN $(3,2,1)$ & Logsig5 & 0.98 & 0.95 & 3.5 \\
ANN(3,3,1) & Tansig1 & 0.91 & 0.90 & 7.9 \\
ANN(3,5,1) & Tansig5 & 0.96 & 0.94 & 5.2
\end{tabular}

\section{Results and discussion}

Concrete technology research is usually intense and long-term experimental studies as well as long-term economic liability for material. The adverse effects of radiation on human health are a known fact. Therefore artificial intelligence programs in high-availability are an alternative solution.

In this study, wall thickness for using in primary radiation shielding was determined in different energy ranges using tenth value layer by ANN. Radiation energy values, TVL and negative logarithm of transmission factor $(n)$ were selected as input parameters and wall shielding thickness values selected as output parameters. Consequently, developed artificial neural networks model outputs were compared with experimental results and it was seen that the results were harmonious. Based on these results of experimental studies of radiation with the aim of overcoming the difficulties of using the methods of neural networks was seen to be useful.

\section{References}

[1] National Council on Radiation Protection and Measurements, Structural Shielding Design and Evaluation for Megavoltage X- and Gamma-Ray Radiotherapy Facilities, Report 151, J. Radiol. Prot. 26, 349 (2006)

[2] D. Sayala, US Radiology 1, 87 (2008).

[3] J.P. Biggs, in: Radiation Shielding for Megavoltage Photon Therapy Machines 52nd Annual Meeting, AAPM, Philadelphia 2010, p. 5.

[4] E. Öztemel, Artificial Neural Networks, Papatya Publishing, Istanbul 2003.

[5] Ö. Terzi, S. Önal, Afr. J. Agricult. Res. 7, 1317 (2012) 